\title{
Unsteady residual distribution schemes for transition prediction
}

\author{
F. Meseguer, E. Valero , C. Martel, J.M. Vega, I.E. Parra \\ School of Aeronautics, Universidad Politécnica de Madrid, Pza. Cardenal Cisneros 3, E-28040 Madrid, Spain
}

A R T I C L E I N F O

Keywords:

Unsteady numerical simulation

Finite element methods

Boundary layer transition

\begin{abstract}
A B S T R A C T
In this work, the unsteady simulation of the Navier-Stokes equations is carried out by using a Residual Distribution Schemes (RDS) methodology. This algorithm has a compact stencil (cell-based computations) and uses a finite element like method to compute the residual over the cell. The RDS method has been successfully proven in steady Navier-Stokes computation but its application to fully unsteady configurations is still not closed, because some of the properties of the steady counterpart can be lost. Here, we proposed a numerical solution for unsteady problems that is fully compatible with the original approach. In order to check the method, we chose a very demanding test case, namely the numerical simulation of a Tollmien-Schlichting (TS) wave in a 2D boundary layer. The evolution of this numerical perturbation is accurately computed and checked against theoretical results.
\end{abstract}

\section{Introduction}

Unsteady flows appear in a wide variety of industrial problems, such as detached flow in high lift aircraft take-off configurations, flow in gaps, transitional flows, flow in very complex shapes imposed by ice accretion, aeroelastic coupling, and buffet prediction. The accurate prediction of these flows is not possible with conventional steady solvers and unsteady simulation is unavoidable.

However, simulation of unsteady flows is generally more computational demanding than its steady counterpart. The steady solvers are designed to have certain numerical properties which tend to either eliminate or dissipate any "non-steady" component of the flow. Therefore, literal translation of these numerical methods by taking a global time step generates very inefficient, highly dissipative solvers, which require a very low time-step to get the desired accuracy. Moreover, some of the numerical properties of the original steady solvers, such as multidimensional upwinding or numerical precision, can be lost in this translation procedure.

In this work, the unsteady simulation of the Navier-Stokes equations is carried out by using a Residual Distribution Schemes (RDS) methodology (Valero et al. [21]). This algorithm has a compact stencil (cell-based computations) and compute the residual over the cell using a finite element method. RDS were initially developed for the solution of scalar advection equations and subsequently extended to systems of equations (Deconinck and Ricchiuto [6]). Regardless of the temporal scheme used, the original RDS formulation cannot be more than first order accurate in timedependent computations due to the inconsistency of the spatial discretization (Ferrante and Deconinck [8]). Various solutions have been proposed to overcome this difficulty; see, e.g., Ferrante and Deconinck [8] and Caraeni [2], who made use of a finite-element like mass-matrix that were consistent with the spatial discretization, and Ricchiuto et al. [16] and Csik and Deconinck [4], who introduce space-time residual distribution schemes. But, all of these papers are focused on inviscid unsteady solutions, with non-steady viscous computation not conclusive (Caraeni et al. [3]) and putting more emphasis on the numerical aspect of the method than in its real predictive capabilities for complex viscous flows. Here, we propose the application of RDS to general systems of conservation laws and, particularly, to non-steady viscous problems.

The performance of this new implementation is tested by the numerical simulation of a Tollmien-Schlichting (TS) wave in a 2D boundary layer. The first stage of the transition process in a flatplate boundary layer is governed by the amplification of TS twodimensional disturbances. The growth or decay of these waves is well described by linear stability theory (Schlichting and Gersten [18]), which provides the temporal and spatial amplification rates of the disturbances under the assumption of parallel flow. Disturbances evolve downstream, with amplitudes growing or decaying as the distance from the leading edge increases.

Various approaches can be found in the literature to numerically compute these perturbations. Most of them consider incompressible flows, either using a linearized version of the NS equations (Theofilis [20]) or integrating the full NS system (Fasel and Konzelmann [7]). However, the direct computation of the boundary layer using the Navier-Stokes equations does not exactly reproduce the linear stability of parallel flows, since non-parallel effects are taken into account. The evaluation of non-parallel effects for incompressible flows has been discussed by different authors, such as Fasel and Konzelmann [7], Saric and Nayfeh [17], and Gaster [9]. Although some differences are observed, they conclude that the 
amplitude and phase distribution with respect to the normal direction $(y)$ are practically coincident with those obtained from the eigenfunctions of linear (parallel) stability theory. These authors also advise that non-parallel effects are strongest close to the wall. These conclusions will be considered in our numerical simulation, but even with this limitations, accurate computation of a physical disturbance is a numerical challenge and as such, it can be considered as a good test for evaluating our method.

The paper is organized as follows. After formulating the problem in the following section, the numerical tool used to calculate the unsteady flow is described in Section 3. The analytical foundations of the TS waves is described briefly in Section 4. The description of the perturbation problem used as validation tool is exposed in Section 5. The validation results are summarized and discussed in Section 6. The paper ends with some summarizing comments at Section 7.

\section{Problem formulation. Navier-Stokes compressible flow}

The governing equations for the compressible, viscous fluid flow are the conservation of mass, momentum, and energy. In nondimensional form, using Cartesian tensor notation, such equations are

$$
\begin{aligned}
& \frac{\partial \rho}{\partial t}+\frac{\partial\left(\rho u_{j}\right)}{\partial x_{j}}=0 \\
& \frac{\partial\left(\rho u_{i}\right)}{\partial t}+\frac{\partial\left(\rho u_{i} u_{j}+p \delta_{i j}\right)}{\partial x_{j}}=\frac{\partial \tau_{i j}}{\partial x_{j}}, \quad i=1,2 \\
& \frac{\partial(\rho e)}{\partial t}+\frac{\partial\left[(\rho e+p) u_{j}\right]}{\partial x_{j}}=-\frac{\partial q_{j}}{\partial x_{j}}+\frac{\partial\left(\tau_{i j} u_{i}\right)}{\partial x_{j}}
\end{aligned}
$$

where $\rho, u_{j}$, and $p$ denote the density, velocity components, and pressure, respectively. The total energy $\rho e$, the viscous stress tensor $\tau_{i j}$, and the heat flux components $q_{j}$ are given by

$$
\begin{aligned}
\rho e & =\frac{p}{\gamma-1}+\frac{1}{2} \rho u_{k} u_{k} \\
\tau_{i j} & =\frac{\mu}{\operatorname{Re}}\left(\frac{\partial u_{i}}{\partial x_{j}}+\frac{\partial u_{j}}{\partial x_{i}}-\frac{2}{3} \frac{\partial u_{k}}{\partial x_{k}} \delta_{i j}\right) \\
q_{j} & =-\frac{\mu}{(\gamma-1) \operatorname{Re} \operatorname{Pr} M^{2}} \frac{\partial T}{\partial x_{j}}
\end{aligned}
$$

The non-dimensional parameters appearing in the above equations are the Reynolds number, Re $=\hat{\rho}_{0} \hat{u}_{0} \hat{L}_{0} / \hat{\mu}_{0}$, the Prandtl number, $\operatorname{Pr}=\hat{\mu}_{0} C_{p} / \hat{k}$, the Mach number, $M=\hat{u}_{0} / \sqrt{(\gamma-1) C_{p} \hat{T}_{0}}$, and the heat capacity ratio, $\gamma=C_{p} / C_{v}$, which are based on the reference density $\left(\hat{\rho}_{0}\right)$, velocity $\left(\hat{u}_{0}\right)$, length $\left(\hat{L}_{0}\right)$, temperature $\left(\hat{T}_{0}\right)$, and molecular viscosity $\left(\hat{\mu}_{0}\right) . C_{p}$ and $C_{v}$ are the constant-pressure and constant-volume specific heat capacities, respectively. On the other hand, the viscosity is temperature dependent and assumed to follow the Sutherland law, which is written in non-dimensional terms as

$$
\mu(T)=\frac{\mu(\hat{T})}{\hat{\mu}_{0}}=\frac{T^{3 / 2}(1+s)}{T+s}
$$

where $T=\hat{T} / \hat{T}_{0}$. The parameter $s$ depends on the reference temperature, e.g., $s=0.37$ for $\hat{T}_{0}=300 \mathrm{~K}$. An equation of state is needed to close the above system, namely

$p=\frac{\rho T}{\gamma M^{2}}$

All numerical computations below will be performed for $\mathrm{Re}=$ $10^{5}, \gamma=1.4$, and various values of the Mach number, using the following reference units for non-dimensionalization: $\hat{u}_{0}=347.37$.
$M \mathrm{~m} / \mathrm{s}, \hat{\rho}_{0}=1.1609 \mathrm{~kg} / \mathrm{m}^{3}, \hat{L}_{0}=(\operatorname{Re} / 2.184 \cdot M) 10^{-7} \mathrm{~m}$, and $\hat{T}_{0}=$ $300 \mathrm{~K}$.

\section{Numerical method}

As anticipated above, the RDS method was initially developed for the solution of scalar advection problems, and then successfully extended to systems of equations, see, e.g., Paillere [14] for a detailed description of the fundamentals of these schemes. Here, we introduce the application of RDS to non-steady problems that result from general non-linear systems of conservation laws.

For illustration, we consider the compressible Navier-Stokes system (1) in two-dimensions, although its generalization to threedimensional systems is straightforward. The system (1) can be written as

$\frac{\partial \mathbf{V}}{\partial t}+\nabla \cdot \mathbf{F}=0$

where $\mathbf{V}(\mathbf{x}, t)$ is the 4 component state vector containing the conserved quantities $\rho, \rho u_{1}, \rho u_{2}$, and $\rho e$, and $\mathbf{F}$ is the $2 \times 4$ flux vector of components $\mathbf{F}_{1}$ and $\mathbf{F}_{2} . \mathbf{F}$ is decomposed in its viscous and non-viscous parts, $\mathbf{F}_{\mathbf{v}}$ and $\mathbf{F}_{\mathbf{n v}}$, respectively, which receive a different numerical treatment.

\subsection{Numerical treatment of non-viscous fluxes}

For $j=1,2$, let $\mathbb{A}_{j}$ be the components of the Jacobian of $\left(\mathbf{F}_{\mathrm{nV}}\right)$. The associated quasi-linear flux is written as

$\nabla \cdot \mathbf{F}_{\mathbf{n v}}=\mathbb{A}_{1} \frac{\partial \mathbf{V}}{\partial x}+\mathbb{A}_{2} \frac{\partial \mathbf{V}}{\partial y}$

In the framework of RDS, the solution is discretized on P1 finite element meshes, which are triangles $(T)$ in 2D in which a piecewise linear solution representation of the solution $\mathbf{V}^{h}$ is sought, namely

$\mathbf{V}^{h}=\sum_{i=1}^{N} \mathbf{V}_{i}\left(\mathbf{x}_{i}, t\right) N_{i}^{h}(\mathbf{x})$

where $N$ is the total number of nodes in the mesh; $\mathbf{V}_{i}\left(\mathbf{x}_{i}, t\right)$ is the time dependent nodal value of the solution at node $i$; and $N_{i}^{h}(\mathbf{x})$ is the piecewise linear shape function that equals one at node $i$ and vanishes outside the elements sharing $i$ as a vertex.

The application of RDS to vectorial schemes is done in the following way. First, integration of (6) over a triangle $T$ yields the definition of the cell residual $\boldsymbol{\Phi}^{T}$,

$\boldsymbol{\Phi}_{n v}^{T}=\int_{T} \nabla \cdot \mathbf{F}_{\mathbf{n v}} d \Omega=\int_{T}\left(\mathbb{A}_{1} \frac{\partial \mathbf{V}}{\partial x}+\mathbb{A}_{2} \frac{\partial \mathbf{V}}{\partial y}\right) d \Omega$

whose discrete form is

$\boldsymbol{\Phi}_{n v}^{T}=\left(\overline{\mathbb{A}}_{1} \frac{\overline{\partial \mathbf{V}}}{\partial x}+\overline{\mathbb{A}}_{2} \frac{\overline{\partial \mathbf{V}}}{\partial y}\right) S_{T}=\sum_{i=1}^{3} \mathbb{K}_{i} \mathbf{V}_{i}$

Due to the fact that $\mathbf{V}$ varies linearly over $T$, its gradient exhibits constant components $\left(\frac{\overline{\partial \mathbf{V}}}{\partial x}\right.$ and $\left.\frac{\overline{\partial \mathbf{V}}}{\partial y}\right)$ in each element, and takes the simplified form $\nabla \mathbf{V}=(1 / 2) \sum_{i=1,3} \mathbf{V}_{i} \mathbf{n}_{i}$, where $\mathbf{n}_{i}=$ $\left(n_{i x}, n_{i y}\right)$ is the inward normal of the face opposite to node $i$, scaled with the area of the element $S_{T}$, and the index $i$ goes through the local nodes of the element $T$. Therefore, the multidimensional inflow matrix reads $\mathbb{K}_{i}=\left(\overline{\mathbb{A}}_{1} n_{i x}+\overline{\mathbb{A}}_{2} n_{i y}\right) / 2$, where $\overline{\mathbb{A}}_{1}$ and $\overline{\mathbb{A}}_{2}$ are the mean value flux Jacobian over the domain $T$.

The hyperbolic nature of (5) implies that the matrix $\mathbb{K}_{i}$ has a set of $q$ real eigenvalues $\left(\Lambda_{i}\right)$ with linearly independent right and left eigenvectors $\left(\mathbb{R}_{i}, \mathbb{L}_{i}\right)$. Therefore, it admits the Jordan decomposition 


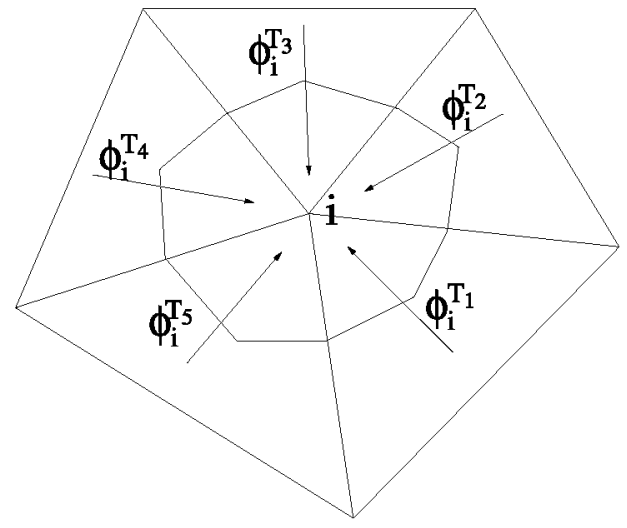

Fig. 1. Schematic representation of RDS approach. Node $i$ is updated from information coming of surrounding cells.

$\mathbb{K}_{i}=\mathbb{R}_{i} \Lambda_{i} \mathbb{L}_{i}, \quad \sum_{i=1}^{3} \mathbb{K}_{i}=0$

Introducing $\Lambda_{i}^{ \pm}=\left(\Lambda_{i} \pm\left|\Lambda_{i}\right|\right) / 2$, the multidimensional upwinding matrices can be defined as

$\mathbb{K}_{i}^{ \pm}=\mathbb{R}_{i} \Lambda_{i}^{ \pm} \mathbb{L}_{i}, \quad i=1,3$

The RDS consists of distributing the residual $\boldsymbol{\Phi}_{n v}^{T}$ to the surrounding nodes, with weights summing up to one for consistency (Fig. 1). The sub-residuals are defined as $\boldsymbol{\Phi}_{i}^{T}$ and

$\boldsymbol{\Phi}_{n v}^{T}=\sum_{i=1}^{3} \boldsymbol{\Phi}_{i}^{T}$

in each triangle $T$. The multidimensional upwinding property distributes the residual to node $i$ (belonging to the triangle $T$ ), with weights proportional to the eigenvalues of the local matrix $\mathbb{K}_{i}^{+}$. A family of residual distribution schemes can be defined to obtain the nodal value $\boldsymbol{\Phi}_{i}^{T}$ (see for instance Paillere [14]). In this work, the Lax-Wendroff (LW) matrix distribution scheme has been considered. In such a case, the distribution of the residual is given by

$\boldsymbol{\Phi}_{i}^{T}=\mathbb{B}_{i} \boldsymbol{\Phi}_{n v}^{T}$

where $\mathbb{B}_{i}$ is the cell-wise constant distribution matrix

$\mathbb{B}_{i}=\frac{1}{3} \mathbb{I}+\frac{1}{2} \nu_{T} \mathbb{K}_{i}\left(\sum_{j \in T} \mathbb{K}_{j}^{+}\right)^{-1}$

that satisfies $\sum_{i=1}^{N} \mathbb{B}_{i}=\mathbb{I}$, where $\mathbb{I}$ is the identity matrix. The parameter $v_{T}$ plays the role of a numerical viscosity. If neglected, the LW scheme is equivalent to a central scheme, which is unstable for compressible flows. High values of $v_{T}$ stabilize the numerical scheme, but introduce excessive numerical dissipation. In this work, the value $v_{T}=0.2$ has been taken as a good compromise between these opposite effects.

As compared to other high-order schemes, one of the main advantages of the RDS methods is that matrix $\mathbb{B}_{i}$ can be modified to satisfy certain properties of monotonicity and accuracy, maintaining the same compact stencil. Therefore, other numerical schemes (such as Low Diffusion-LDA and Narrow-N schemes) can be used that exhibit better monotonicity properties. However, they are also more dissipative. For the particular case of boundary layer stability, LW scheme is a good compromise between stability and low-dissipative behavior.

After Abgrall [1], it is known that RDS schemes are well posed, even if the matrix $\left(\sum_{j \in T} \mathbb{K}_{j}^{+}\right)^{-1}$ is singular.

\subsection{Numerical treatment of viscous fluxes}

The contribution of the viscous fluxes $\left(\mathbf{F}_{\mathbf{v}}\right)$ can be obtained using a finite element approximation. This is obtained multiplying the viscous component of the second term of (5) by the test function $\omega_{i}^{h}$ (see (14) below) with compact support $\Omega_{i}$ (the region defined by all triangles meeting node $i$ ), and integrating by parts. It follows that

$$
\begin{aligned}
\boldsymbol{\Phi}_{v}^{T} & =\int_{\Omega_{i}} \omega_{i}^{h} \nabla \cdot \mathbf{F}_{\mathbf{v}} d \Omega=-\int_{\Omega_{i}} \nabla N_{i}^{h} \cdot \mathbf{F}_{\mathbf{v}} d \Omega \\
& =-\sum_{T} \int_{T} \frac{\mathbf{n}_{i}}{2 S_{T}} \cdot \mathbf{F}_{\mathbf{v}} d \Omega=-\sum_{T} \frac{\mathbf{n}_{i}}{2} \cdot \overline{\mathbf{F}}_{\mathbf{v}}
\end{aligned}
$$

where the contribution of the boundary of $\Omega_{i}$ has been canceled because of the compact support of $\omega_{i}$ and $\overline{\mathbf{F}}_{\mathbf{v}}$ is an average value of the flux $\mathbf{F}_{\mathbf{v}}$ on the element $T$.

\subsubsection{Temporal discretization}

In order to perform the time integration, an equivalent PetrovGalerkin (PG) formulation is used that is consistent with the spatial discretization of the residual distribution. The PG weighting function $\omega_{i}$ is defined as equal to the shape function plus and upwinding correction term $\alpha_{i}$, namely

$\omega_{i}^{h}=N_{i}^{h} \mathbb{I}+\alpha_{i} P^{e}$

where $P^{e}=1$ on the element subdomain and zero elsewhere Then, the finite element spatial discretization of (5) is written as

$\sum_{T} \int_{T} \omega_{i}^{h}\left[\frac{\partial \mathbf{V}}{\partial t}+\left(\mathbb{A}_{1} \frac{\partial \mathbf{V}}{\partial x}+\mathbb{A}_{2} \frac{\partial \mathbf{V}}{\partial y}\right)+\nabla \cdot \mathbf{F}_{\mathbf{v}}\right] d \Omega=0$

with the sum extended to all the elements $(T)$ surrounding node $i$.

Setting $\mathbb{A}_{i}=\overline{\mathbf{A}}_{i}$ (which is constant over each element $T$ ) and recalling the definition of the sub-residuals (9)-(13), yields

$\sum_{T} \int_{T} \omega_{i}^{h} \frac{\partial \mathbf{V}}{\partial t} d \Omega+\sum_{T} \frac{\boldsymbol{\Phi}_{n v}^{T}}{S_{T}} \int_{T} \omega_{i}^{h} d \Omega+\boldsymbol{\Phi}_{v}^{T}=0$

Here, both the viscous and non-viscous sub-residuals, $\boldsymbol{\Phi}_{v}^{T}$ and $\boldsymbol{\Phi}_{n v}^{T}$, have been taken out from integral because of the linear representation of the conservative variables. Thus, the distribution matrix $\mathbb{B}_{i}$ (11) can be expressed as

$\mathbb{B}_{i}=\frac{1}{S_{T}} \int_{T} \omega_{i}^{h} d \Omega=\frac{1}{S_{T}}\left[\int_{T} N_{i}^{h} \mathbb{I} d \Omega+\alpha_{i} S_{T}\right]$

where, in order to satisfy the equivalence between the second term of (16) and (11), the correction term $\alpha_{i}$ must be defined as

$\alpha_{i}=\mathbb{B}_{i}-\frac{1}{S_{T}} \int_{T} N_{i}^{h} \mathbb{I} d \Omega$

For a Galerkin finite element discretization, the second term of the right-hand side can be identified as the distribution matrix of a Galerkin (central) discretization

$\mathbb{B}_{i, \mathrm{Gal}}=\frac{1}{S_{T}} \int_{T} N_{i}^{h} d \Omega=\frac{1}{3} S_{T} \mathbb{I}$

where the last term stands for triangular elements and linear shape functions, which implies that

$\alpha_{i}=\mathbb{B}_{i}-\mathbb{B}_{i, \text { Gal }}$ 
Using this definition of $\alpha_{i}$ and (7), the spatial semi-discretization (15) can be written as

$\sum_{T} \sum_{j} m_{i j}^{T} \frac{d \mathbf{V}_{j}}{d t}=-\sum_{T} \mathbb{B}_{i} \boldsymbol{\Phi}_{T}+\boldsymbol{\Phi}_{v}^{T}$

with the mass matrix defined as

$m_{i j}^{T}=\int_{S_{T}} \omega_{i}^{h} N_{j}^{h} d \Omega$

After assembling the global mass matrix, a system of ordinary differential equations is obtained, namely

$\mathbb{M} \frac{d \mathbf{U}}{d t}+\mathbf{R}(\mathbf{U})=0, \quad \mathbf{U}=\left\{\mathbf{V}_{0} \cdots \mathbf{V}_{N}\right\}$

The numerical integration of this system can be made considering the two-parameter discretization schemes

$\frac{(1+\xi) \mathbb{M}^{n+1} \Delta \mathbf{U}^{n+1}-\xi \mathbb{M}^{n} \Delta \mathbf{U}^{n}}{\Delta t}=-\left[\theta \mathbf{R}\left(\mathbf{U}^{n+1}\right)+(1-\theta) \mathbf{R}\left(\mathbf{U}^{n}\right)\right]$

where $\Delta \mathbf{U}^{n+1}=\mathbf{U}^{n+1}-\mathbf{U}^{n}$ is the increment of the variables at each time step $\Delta t$. These scheme are second order accurate in time provided that

$\theta=\xi+\frac{1}{2}$

After some calibration that focussed on stability properties, the backward scheme $(\xi=1 / 2, \theta=1)$ has been selected, namely

$\frac{3}{2} \mathbb{M}^{n+1} \Delta \mathbf{U}^{n+1}-\frac{1}{2} \mathbb{M}^{n} \Delta \mathbf{U}^{n}=-\Delta t \mathbf{R}\left(\mathbf{U}^{n+1}\right)$

Calibration consisted on numerical experiments, which have shown that this scheme gives fairly stable results and shows a quite small numerical viscosity in fairly long time spans. In other words, the selected scheme is A-stable and gives better results when the imaginary parts of the eigenvalues of the Jacobian go to infinity.

Finally, in order to solve Eq. (18) (which is non-linear in the unknown $\mathbf{U}^{n+1}$ ) at each time step, a Newton sub-iteration is applied, which usually provides $\mathbf{U}^{n+1}$ in three sub-iterations, with a non-steady residual level of $10^{-10}$.

\section{Analytical description of Tollmien-Schlichting waves}

A short analytical description of the compressible boundary layer over a flat plate at zero incidence is now provided that will be used in next section to compare with the numerical simulation of the boundary layer. In the boundary layer approximation, we used the selfsimilar variable

$\delta=\sqrt{x / \operatorname{Re}} \ll 1, \quad \eta=y / \delta$

where $\delta=\sqrt{x / \operatorname{Re}}$ is proportional to the boundary layer thickness, and seek steady states (denoted with the subscript $S$ ) as that only depend on $\eta$, namely

$$
\begin{aligned}
& \left(\rho_{S},\left(u_{1}\right)_{S},\left(u_{2}\right)_{S}, T_{S}, p_{S}\right) \\
& \quad=(\rho(\eta), u(\eta), \delta V(\eta), T(\eta), p(\eta))
\end{aligned}
$$

where the wall-normal velocity component has been also rescaled with $\delta$. For convenience, we consider the Reynolds number based on the boundary layer thickness, namely

$R=\operatorname{Re} \delta \equiv \sqrt{x \operatorname{Re}} \gg 1$

Replacing (19)-(20) into (1) and neglecting $O(1 / R)$-terms yields the following system of ordinary differential equations $-\eta(\rho u)^{\prime}+2(\rho V)^{\prime}=0, \quad \rho T=\mathcal{B}_{1} p$

$\rho(-\eta u+2 V) u^{\prime}=2\left[\mu u^{\prime}\right]^{\prime}$

$\rho(-\eta u+2 V) T^{\prime}=2 \operatorname{Pr}^{-1}\left[\mu T^{\prime}\right]^{\prime}+2 \mathcal{B} \mu\left(u^{\prime}\right)^{2}$

where primes denote derivation with respect to $\eta$ and the parameters $\mathcal{B}_{1}$ and $\mathcal{B}$ are defined as $\mathcal{B}_{1}=\gamma M^{2}$ and $\mathcal{B}=(\gamma-1) M^{2}$. The boundary conditions are

$u=v=T^{\prime}=0 \quad$ at $\eta=0, u=1, \quad T=T_{e} \quad$ at $\eta=\infty$

which correspond to an outer flow temperature $T_{e}$ and a thermally insulated wall. Namely, we assume that the steady state is reached after a transient in which thermal equilibrium between the solid and the surrounding air is established.

The TS-modes of this self-similar steady state exhibit a streamwise wavelength that is about one order of magnitude larger than the local boundary-layer thickness, and either grow or decay exponentially in a characteristic length comparable to its wavelength; thus, they can grow quite fast as to promote non-linear interactions. The mathematical description of TS-modes is obtained introducing a perturbation over the previous steady solution, namely

$$
\begin{aligned}
\left(\rho, u_{1}, v_{1}, T, p\right)= & (\rho, u, \delta V, T, p) \\
& +\left[(r, \phi, \delta \psi, \theta, \pi) \mathrm{e}^{\mathrm{i}(\alpha x-\omega t) / \delta}+\text { c.c. }\right]
\end{aligned}
$$

where c.c. stands for the complex conjugate. In the analysis, the temporal $(\omega)$ and spatial $(\alpha)$ amplification rates have been rescaled with $\delta$. The amplitudes of the modes $(r, \phi, \delta \psi, \theta, \pi)$ are assumed to depend only on $\eta$. Substituting the above expression into (1) and linearizing, we obtain

$$
\begin{aligned}
& \mathrm{i}(\alpha u-\omega) r+(\rho \psi)^{\prime}+\mathrm{i} \alpha \rho \phi=0, \quad \mathcal{B}_{1} \pi=\mathrm{Tr}+\rho \theta \\
& 3 \mathrm{i} \rho(\alpha u-\omega) \phi+3 \rho u^{\prime} \psi \\
& =-3 \mathrm{i} \alpha \pi+R^{-1}\left[3\left(\mu \phi^{\prime}\right)^{\prime}+\mu\left(\mathrm{i} \alpha \psi^{\prime}-4 \alpha^{2} \phi\right)\right. \\
& \left.\quad+3 \mathrm{i} \alpha \mu^{\prime} T^{\prime} \psi+3\left(\mu^{\prime} u^{\prime} \theta\right)^{\prime}\right] \\
& 3 \mathrm{i} \rho(\alpha u-\omega) \psi \\
& \quad-3 \pi^{\prime}+R^{-1}\left[4\left(\mu \psi^{\prime}\right)^{\prime}+\mu\left(\mathrm{i} \alpha \phi^{\prime}-3 \alpha^{2} \psi\right)\right. \\
& \left.\quad+\mathrm{i} \alpha \mu^{\prime}\left(-2 T^{\prime} \phi+3 u^{\prime} \theta\right)\right] \\
& \mathrm{i} \rho(\alpha u-\omega) \theta+\rho T^{\prime} \psi \\
& =(\operatorname{Pr} R)^{-1}\left[\left(\mu \theta^{\prime}\right)^{\prime}-\alpha^{2} \mu \theta+\left(\mu^{\prime} T^{\prime} \theta\right)^{\prime}\right]+\mathrm{i} \mathcal{B}(\alpha u-\omega) \pi \\
& \quad+B R^{-1}\left[2 \mu u^{\prime}\left(\phi^{\prime}+\mathrm{i} \alpha \psi\right)+\mu^{\prime}\left(u^{\prime}\right)^{2} \theta\right]
\end{aligned}
$$

where $\mu$ denotes $\mu(T)$. A detailed description of the derivation of these equations for compressible flows can be found in Mack [13].

The appropriate boundary conditions are

$\phi=\psi=\theta^{\prime}=0 \quad$ at $\eta=0, \quad \phi=\psi=\theta=0 \quad$ at $\eta=\infty$

Two remarks are now in order:

- The boundary conditions at $\eta=\infty$ must be numerically imposed at a (large but) finite distance and treated conveniently (Keller [11]), to avoid large errors due to wave-reflection.

- We are retaining small $O(1 / R)$-terms, which account for viscous and heat conduction effects and are essential to trigger the instability that promotes TS waves. The asymptotic analysis of (25)-(26) as $R \rightarrow \infty$ leads to a triple-deck problem [19], which requires to consider fractional powers of $R^{-1}$ and yields a poor approximation. Thus, the usual strategy is to retain $O\left(R^{-1}\right)$-terms, as we do here, and numerically solving the resulting stiff problem. This can be done either discretizing the boundary value problem or, as we have done here, using a 
shooting method combined with a continuous orthonormalization method [5].

\section{Fully non-linear numerical description of the boundary layer problem}

In order to validate the numerical method described in Section 3, we consider the numerical model of the compressible boundary layer of a flat plate at zero incidence, whose linear approximation was provided in Section 4. To this end, we should consider in principle an integration domain whose upstream boundary is fairly close to the leading edge of the plate and extends downstream to a position beyond transition, which arises at fairly high Reynolds namely Re $\sim 10^{5}$. Such computational domain leads to a numerically quite costly process. This is because the smallest scale associated with the viscous sublayer must be described. In order to overcome such a difficulty, we take a computational domain in the streamwise direction covering only a portion of the boundary layer, and impose the steady profile at the entrance. Thus, we consider the computational domain

$x_{0}<x<x_{0}+L, \quad 0<y<y_{0}$

with $L \ll x_{0}$ but somewhat large as to include several wavelengths of the relevant waves, and $y_{0}$ is somewhat large compared to the boundary layer thickness. All lengths are in non-dimensional form.

Then, we calculate the Navier-Stokes steady state solution, $\left(\rho_{s}, u_{1 s}, u_{2 s}, T_{s}\right)$, with the boundary conditions

$$
\begin{aligned}
& \left(u_{1 s}, u_{2 s}\right)=\left(u_{1 b}(y), u_{2 b}(y)\right), \quad \rho_{s}=\rho_{b}(y) \text { at } x=x_{0} \\
& p=1 /\left(\gamma M^{2}\right) \quad \text { at } y=y_{0} \\
& \left(u_{1}, u_{2}\right)=0, \quad \partial T / \partial y=0 \quad \text { at } y=0 \\
& \partial^{2} u_{i} / \partial x^{2}=0, \quad i=1,2 \text { at } x=x_{0}+L
\end{aligned}
$$

where the plate is assumed to be thermally insulated, and $\left(\rho_{b}, \boldsymbol{u}_{1 b}, \boldsymbol{u}_{2 b}\right)$ are the three leading components of the Blasius selfsimilar steady state solution at $x=x_{0}$, given by (20). At $y=y_{0}$ the outflow pressure boundary condition is imposed, whereas a simple extrapolation from interior points is used at $x=x_{0}+L$. This steady initial solution does not coincide exactly with the self-similar Blasius solution (20), due to non-parallel effects.

Once this steady solutions is obtained, the non-steady spatial evolution of a disturbance is studied (see below for a better description). In order to minimize the effects of the boundary on the disturbances, the following considerations have been taken into account (Fasel and Konzelmann [7]):

- At $x=x_{0}+L$, the spurious reflection is minimized using an absorbing boundary layer condition, namely

$$
\frac{\partial^{2} u_{i}^{\prime}}{\partial x^{2}}=-\alpha u_{i}^{\prime}, \quad i=1,2
$$

Here, the prime denotes the disturbance variable, $u_{i}^{\prime}=u_{i s}-$ $u$, and $\alpha$ is the local wavenumber of the disturbance waves. The value of $\alpha$ is taken from the local linear stability analysis solutions (25) without non-parallel correction.

- At $y=y_{0}$, a similar approach is followed. However, here the unsteady disturbance velocity components are assumed to decay exponentially according to the following law

$$
\frac{\partial u_{i}^{\prime}}{\partial y}=-\frac{\alpha}{\operatorname{Re}^{1 / 2}} u_{i}^{\prime}, \quad i=1,2
$$

Additionally, the upper length $y_{0}$ is assumed to be far enough from the wall. According to our computations, the disturbances typically extends around ten displacements thickness to completely disappear. Thus, a value of $y_{0}$ of the order of twenty boundary layer displacements thickness has been used in the computations. Moreover, an unnecessary increase of the number of nodes has been avoided using a potential law to cluster the nodes close to the wall.

With this approach, no spurious reflections have been observed in the computations. The effect of these "inexact" output boundary conditions has been significant only in a maximum of one wavelength of the perturbation upstream of the flow, which in fact limits the valid extension of the computational domain.

In order to generate a TS wave in the boundary layer, we include a time-periodic blowing and suction through a narrow strip at the wall (see, e.g., Fasel and Konzelmann [7] and Theofilis [20]) Thus, a vibrating membrane oscillating with amplitude $\varepsilon$ and frequency $\omega_{1}$ equal to that of the modeled TS wave is included at some position $x=x_{1}$. The size of the membrane is not essential because of the spatially parabolic character of the boundary layer. However, a membrane size similar to the wavelength of the TS wave is convenient to facilitate wave generation. Such a membrane is modeled as

$$
\begin{aligned}
& u_{1}=0, \quad u_{2}(x, t)=\varepsilon f(x) \sin \omega_{1} t \quad \text { if }\left|x-x_{1}\right|<\lambda_{1} / 2 \\
& u_{1}=u_{2}=0 \quad \text { if either } x_{0}<x \leqslant x_{1}-\lambda_{1} / 2 \text { or } \\
& x_{1}+\lambda_{1} / 2 \leqslant x<x_{0}+L
\end{aligned}
$$

where $\omega_{1}$ and $\lambda_{1}$ are respectively the frequency and the wavelength of the perturbation provided by the linear stability analysis; $x_{1}=\lambda_{1}$ is the wall coordinate where the strip is located and $f(x)$ is chosen as

$$
\begin{aligned}
& f(x)=\tilde{f}(\xi), \quad \xi=\frac{x-x_{1}+\lambda_{1} / 2}{\lambda_{1} / 2}, \quad \text { if } x_{1}-\lambda_{1} / 2<x<x_{1} \\
& f(x)=-\tilde{f}(\xi), \quad \xi=\frac{x_{1}+\lambda_{1} / 2-x}{\lambda_{1} / 2}, \quad \text { if } x_{1}<x<x_{1}+\lambda_{1} / 2
\end{aligned}
$$

where

$$
\tilde{f}(\xi)=15.18175 \xi^{5}-35.4375 \xi^{4}+20.25 \xi^{3}
$$

This distribution produces clean localized disturbances and causes negligible time-dependent changes in the mean flow (Konzelmann et al. [12]). In fact, it represents a realistic situation, which is also applicable for large-amplitude calculations. As it was explained in Section 1 , a spatial perturbation approach is followed in this analysis. Taking a fixed perturbation frequency $\omega$, the corresponding spatial evolution rate $\alpha$ is determined. The real part of $\alpha, \alpha_{r}$, defines the streamwise wave number and its imaginary part, $\alpha_{i}$ is the spatial growth rate of the disturbance.

The good numerical properties of RDS (namely, low diffusion, low dissipative effects, second order precision, genuinely multidimensional, and good upwinding properties) make RDS suitable for computing compressible flows. Thus, it is expected that the developed numerical tool must be sufficiently precise to give an accurate description of the TS-modes that are involved in the boundary layer evolution, which exhibit both large frequencies (and wavenumbers) and quite small growth/decay rates.

\section{Verification and validation}

The perturbation problem described in the previous section has been computed for one value of the Reynolds number, $\operatorname{Re}=10^{5}$ and two values of the Mach number, $M=0.3$ and 0.7. According 
Table 1

Characteristics parameters of the computations.

\begin{tabular}{lllc}
\hline Mach & Reynolds & $L_{0}(\mathrm{~m})$ & $u_{0} / L_{0}(\mathrm{~Hz})$ \\
\hline 0.3 & $10^{5}$ & $1.52 \times 10^{-2}$ & 6.827 \\
0.7 & $10^{5}$ & $6.54 \times 10^{-3}$ & 37.174 \\
\hline
\end{tabular}

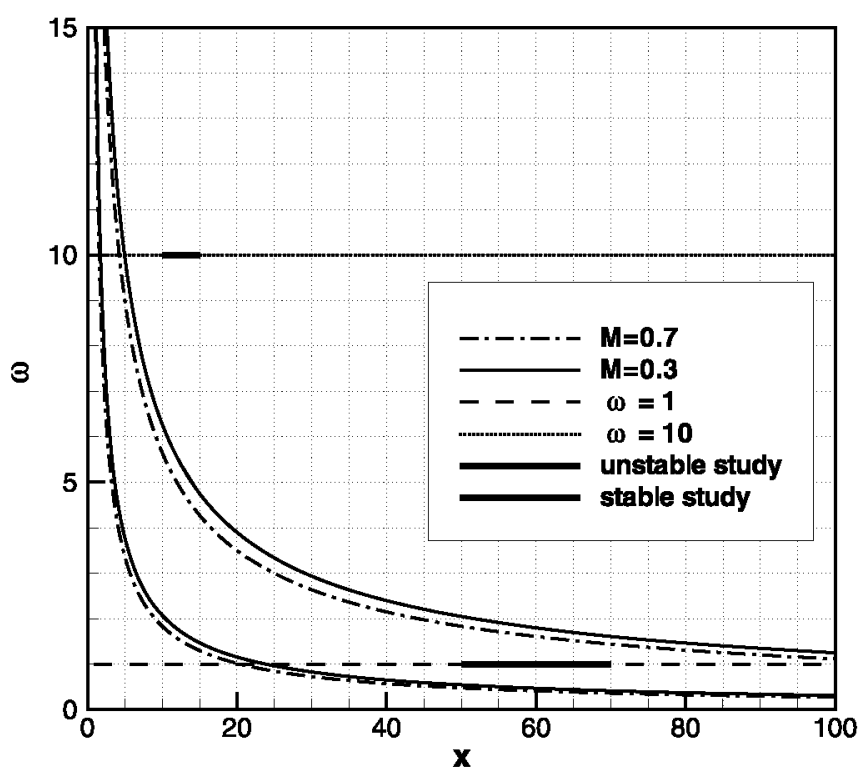

Fig. 2. Neutral stability curves obtained by linear stability theory at Mach num bers 0.3 and 0.7 . The analyzed stable and unstable TS-modes are also indicated.

to our comments at end of Section 2, these two values of $M$ correspond to the streamwise characteristic lengths $L_{0}=1.52 \cdot 10^{-2} \mathrm{~m}$ and $6.54 \cdot 10^{-3} \mathrm{~m}$, respectively. The boundary layer marginal stability curves corresponding to Mach numbers 0.3 and 0.7 are shown in Fig. 2. The horizontal axis is the non-dimensional streamwise coordinate $x$, which is related to the local Reynolds number through Eq. (21), and the vertical axis is $\omega$, the non-dimensional frequency. The reference dimensional values are given in Table 1. Following the perturbation strategy defined in (29), only one TSfrequency $\omega$ is excited at a time, so advancing streamwise means moving in a straight horizontal line in Fig. 2. Two different TSfrequencies have been considered, namely $\omega=1$, which involves a perturbation in the unstable region, and $\omega=10$, which corresponds to the stable region. Some preliminary tests and a detailed convergence analysis have been performed. In order to simplify the exposition these results are shown in Appendix A.

- Unstable frequency: $\omega=1$, lower straight line of Fig. 2. According to linear stability theory, these TS-waves are unstable for local Reynolds numbers $\sqrt{\operatorname{Re} x}$ above 1544 and 1430 , for $M=0.3$ and $M=0.7$, respectively. For the external Reynolds number considered here, $\operatorname{Re}=10^{5}$, the amplification factor $\alpha_{i}$ has been depicted vs. $x$ in Fig. 3. In order to facilitate comparison between the analytical and numerical results, a part of the fluid domain that is close to the maximum amplification rate $(x \in[50,70])$ is selected as computational domain. In that region, $\alpha_{i}$ shows small variations around its maximum value ( 0.27 for $M=0.3$ and 0.23 for $M=0.7$ ). In addition, the non-dimensional wavelength $(\lambda)$ in the area of study (showed in Fig. 4) is roughly constant (1.68 and 1.83 respectively for $M=0.3$ and $M=0.7$ ). Consequently, the chosen computational domain allows for simulating more than 10 wavelengths of the TS-waves. On the other hand, a computational domain thickness of 20 boundary layer thicknesses has been considered in the wall normal direction $\left(y_{0}\right)$. Although the strong

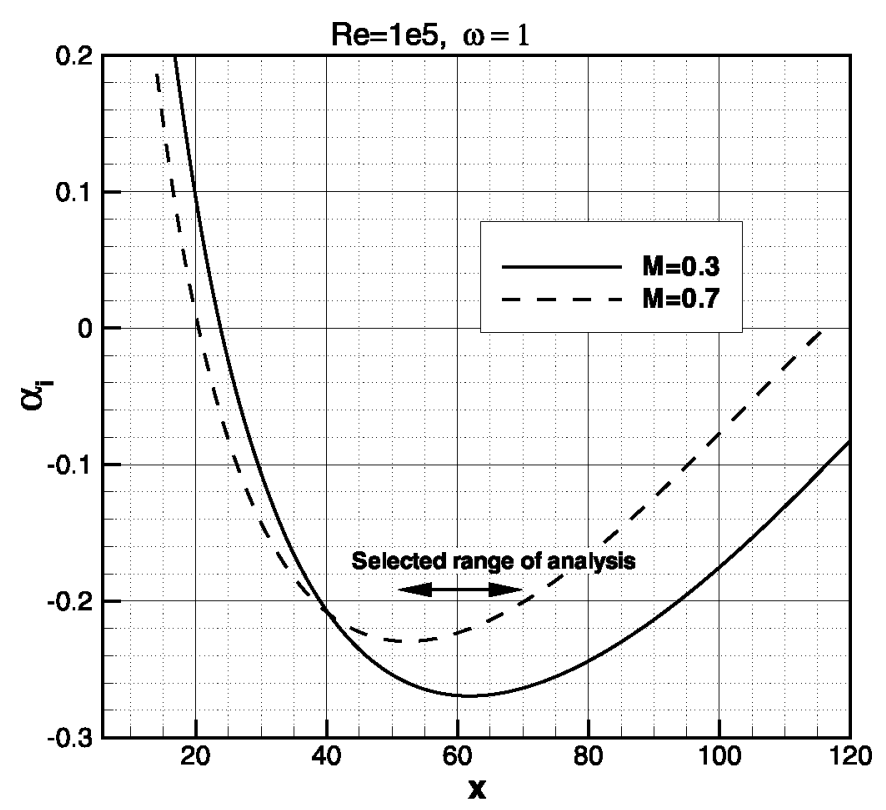

Fig. 3. Evolution of the spatial amplification factor as a function of boundary layer streamwise coordinate $x$ for TS-waves of frequency $\omega=1$.

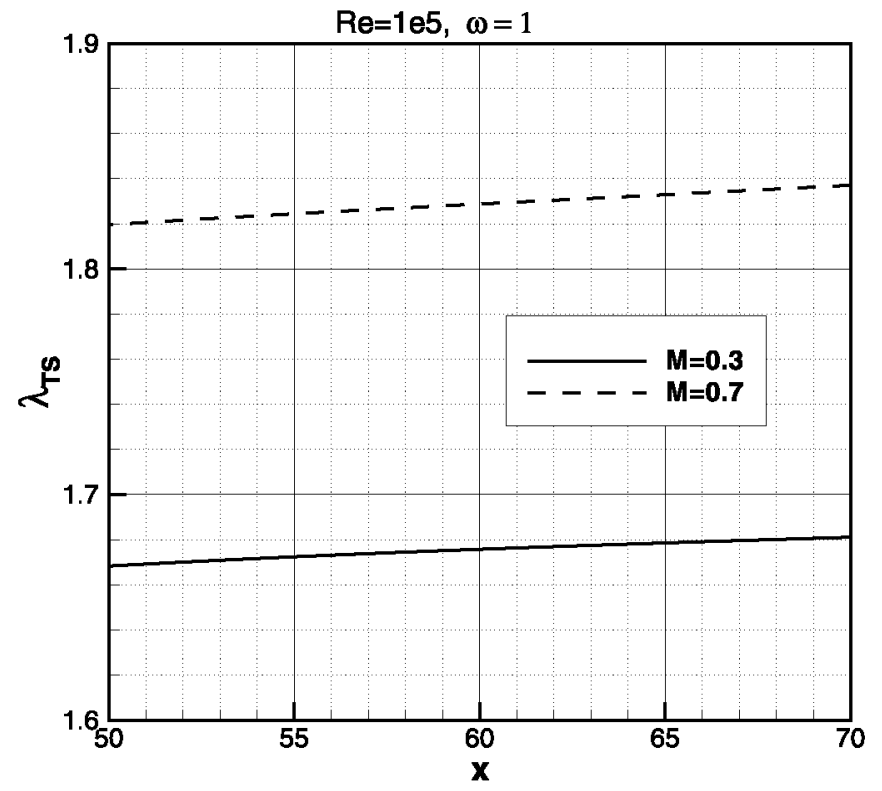

Fig. 4. Evolution of TS wavelength inside the computational domain for unstable TS-waves of frequency $\omega=1$.

variations of the TS wave modes are clearly located inside the boundary layer, a previous sensitivity analysis has shown that the TS waves can extent in some cases to more than 10 boundary layer thicknesses. Thus, to be consistent with the far field normal boundary condition, such an extended computational domain must be considered.

Two kind of comparisons are made between the results from both the full Navier-Stokes integration and linear stability theory. (a) The normalized horizontal and vertical velocity profiles obtained for $M=0.7$ at $x=60$ are compared respectively in Figs. 5 and 6. As already observed in incompressible flows, non-parallel effects are almost negligible in compressible flows and a very good agreement between both numerical and analytical profiles is obtained. (b) The spatial evolution of both the numerical and the analytical amplification factors are com- 


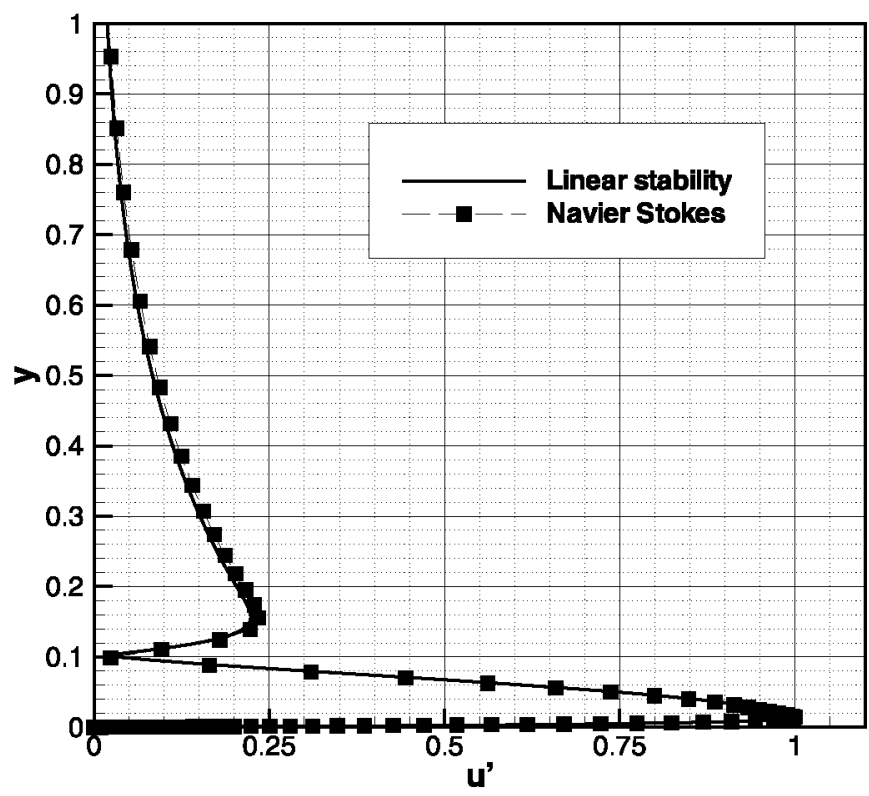

Fig. 5. Comparison between the normalized amplitude distribution of the horizontal velocity at $x=60, \omega=1, \operatorname{Re}=10^{5}, M=0.7$, between the Navier-Stokes computation and linear stability theory.

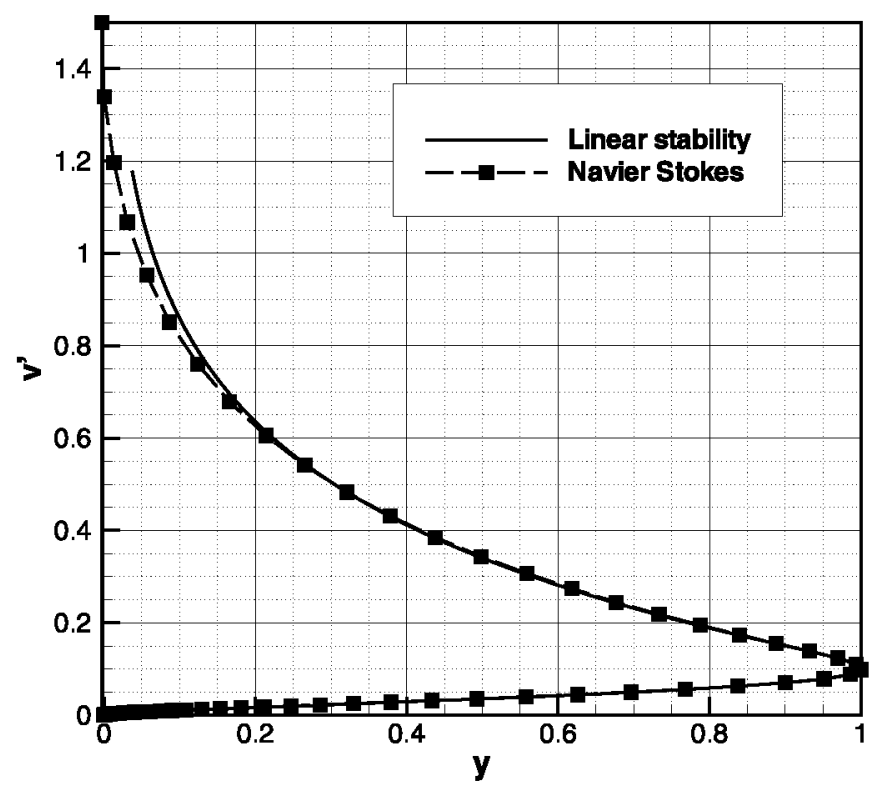

Fig. 6. Comparison between the normalized amplitude distribution of the vertical velocity at $x=60, \omega=1, \operatorname{Re}=10^{5}, M=0.7$, between the Navier-Stokes computation and linear stability theory.

pared in Fig. 7. Numerically, the TS-wave amplification factor $\alpha_{i}$ is computed according to the formula

$\alpha_{i}(x)_{n s}=\log \left(\frac{A(x)}{A_{0}}\right)$

where $A$ is chosen as the maximum value (per wavelength) of the perturbation of streamwise velocity component $u^{\prime}$ (see Fig. 8), and $A_{0}$ is some initial perturbation value taken as reference. The analytical result is obtained by integration of the amplitude factor given by linear stability theory (Fig. 3)

$\alpha_{i}(x)_{l s}=-\int_{x_{0}}^{x} \alpha_{i}(x) d x$

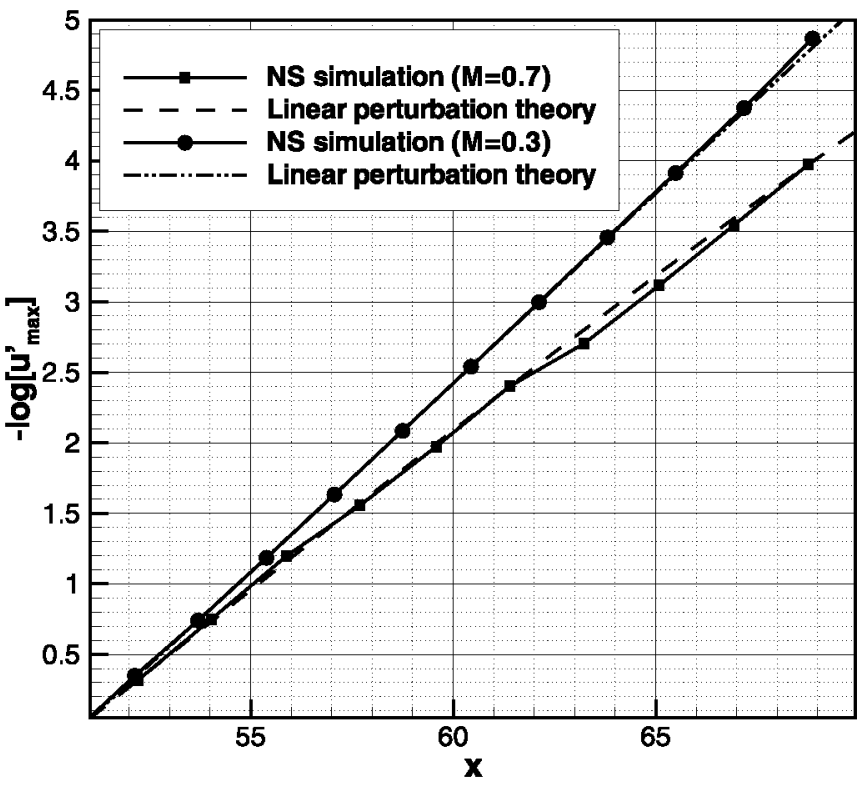

Fig. 7. Numerical comparison between the spatial growth rate between NavierStokes computations and linear stability theory for $\omega=1$ and Mach numbers 0.3 and 0.7 .

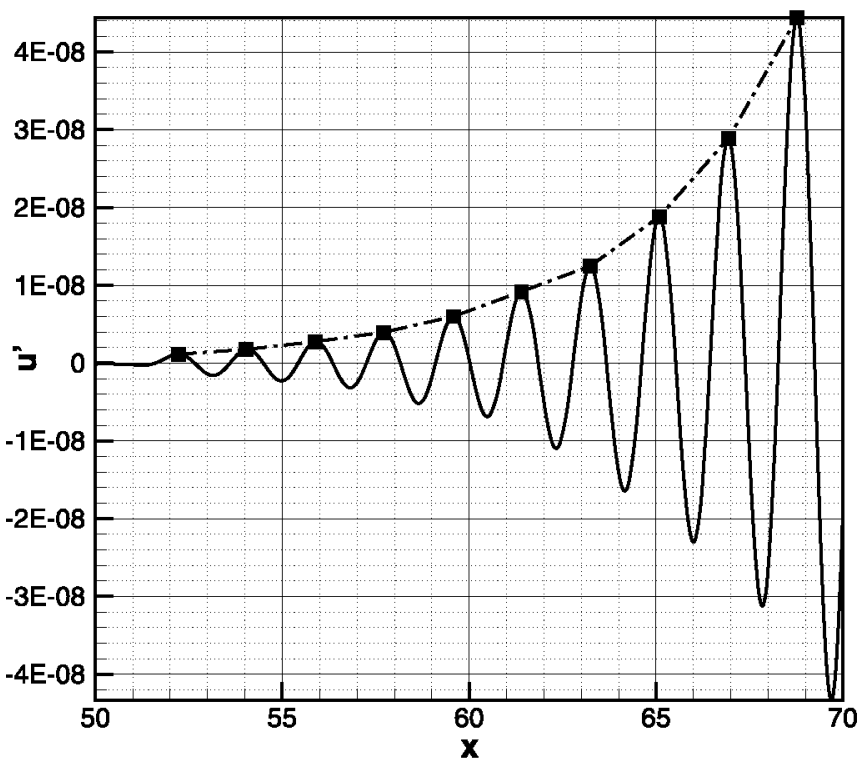

Fig. 8. Maximum values of the perturbation for $\omega=1, \operatorname{Re}=10^{5}, M=0.7, x_{0}=50$ $n x \times n y=500 \times 41$ and 100 steps per period.

Apart from a fairly small oscillation, numerical and analytical results essentially coincide. Additionally, the average values of $-\alpha_{i}$ in the computational domain are shown in Table 2.

- Stable frequency: $\omega=10$, upper straight line in Fig. 2. Now, the TS waves are stable. The streamwise evolution of $\alpha_{i}$ given by linear stability theory is represented in Fig. 9 for local Reynolds numbers $\sqrt{\operatorname{Re} x}=704$ and 652 and $\alpha_{i} \simeq 2.6$ and 2.1, which correspond to $M=0.3$ and $M=0.7$, respectively. In order to minimize the $\alpha_{i}$ variations, the computational domain is restricted to $x \in[10,15]$; the associated non-dimensional wavelengths are also essentially constant, with a small variation around respectively 0.27 and 0.3 (see Fig. 10). Note that the computational domain with $L=5$ contains at least 15 streamwise wavelengths. Finally, as in the case of $\omega=1$, the domain extends up to 20 boundary layer thicknesses in 
Table 2

Average spatial growth rate in the computational domain for $\omega=1$. Comparison between full Navier-Stokes (NS) and linear stability theory (LST).

\begin{tabular}{llll}
\hline Mach & Reynolds & NS & LST \\
\hline 0.3 & $10^{5}$ & -0.268 & -0.270 \\
0.7 & $10^{5}$ & -0.220 & -0.214 \\
\hline
\end{tabular}

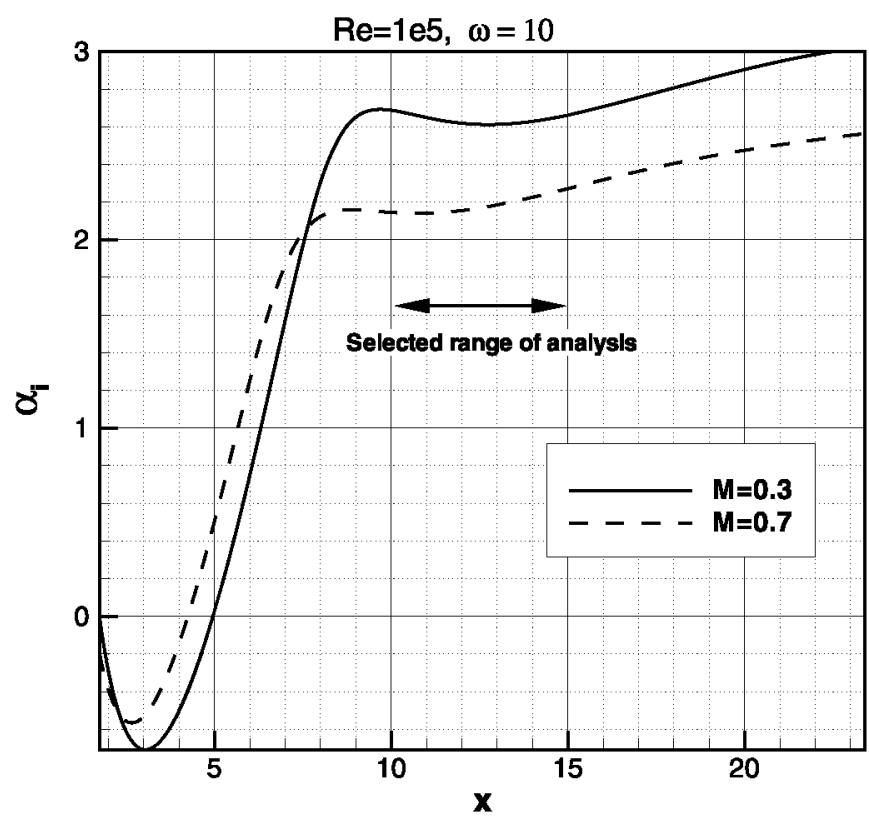

Fig. 9. Evolution of the spatial amplification factor as a function of boundary layer streamwise coordinate $x$ for TS-waves of frequency $\omega=10$.

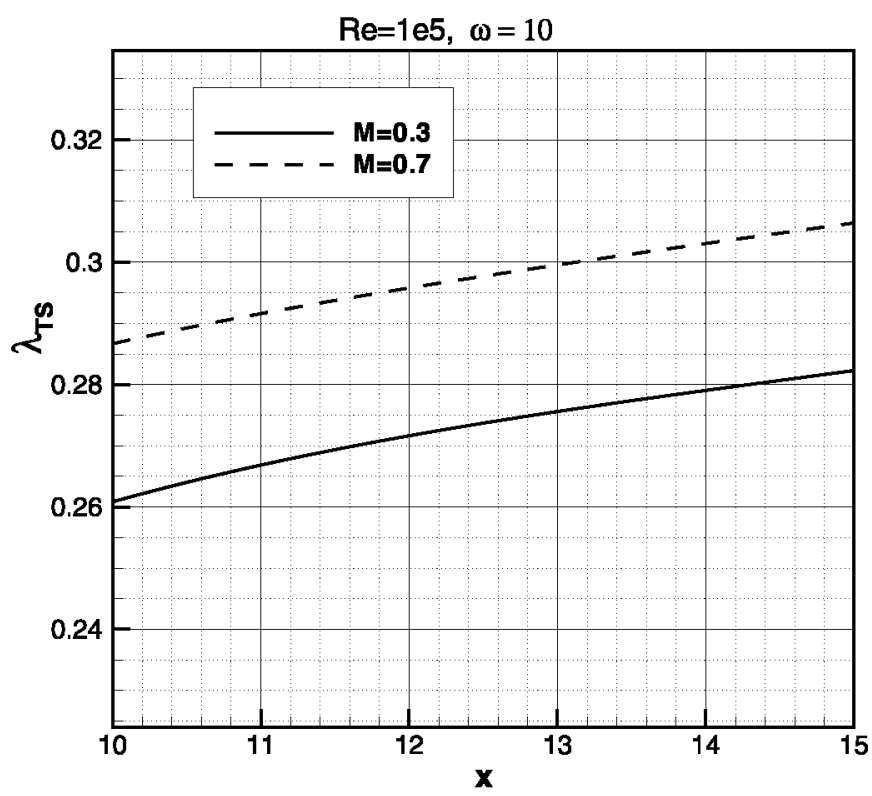

Fig. 10. Evolution of TS wavelength inside the computational domain for unstable TS-waves of frequency $\omega=10$.

the wall normal direction, which corresponds to a height of approximately $y_{0}=0.5$ for both $M=0.3$ and $M=0.7$.

The comparison between the linear stability theory and Navier-Stokes computations (through the formula (31)) is shown in Fig. 11. Again, accurate quantitative and qualitative results are observed. The average values of $-\alpha_{i}$ in the computational domain are shown in Table 3.
Table 3

Average spatial growth rate in the computational domain for $\omega=10$. Comparison between full Navier-Stokes (NS) and linear stability theory (LST).

\begin{tabular}{llll}
\hline Mach & Reynolds & NS & LST \\
\hline 0.3 & $10^{5}$ & 2.674 & 2.641 \\
0.7 & $10^{5}$ & 2.144 & 2.130 \\
\hline
\end{tabular}

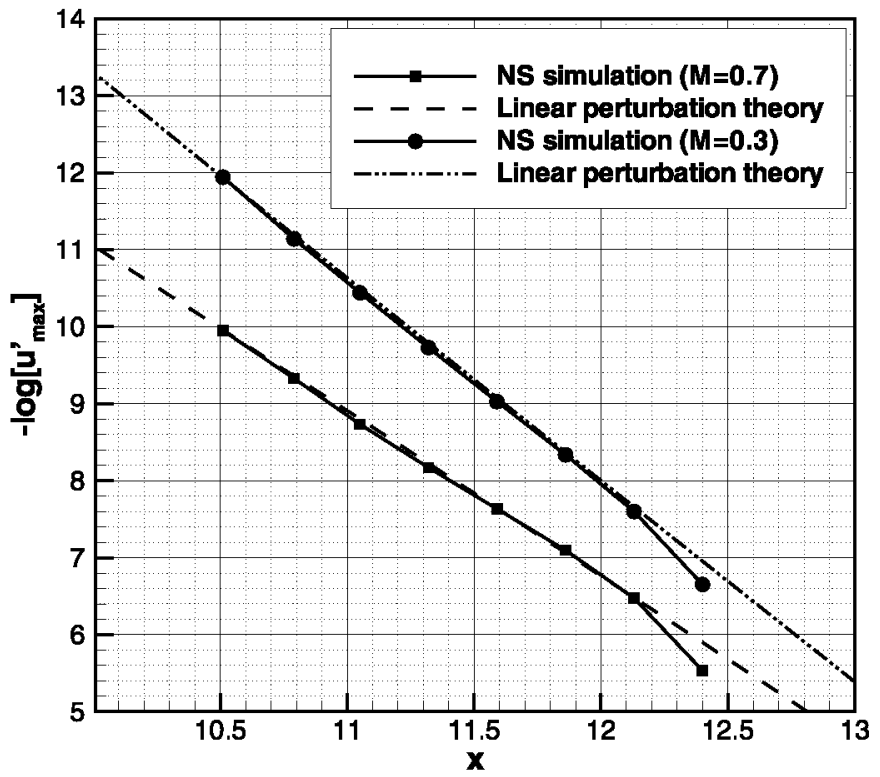

Fig. 11. Numerical comparison between the spatial growth rate between NavierStokes computations and linear stability theory for $\omega=10$ and Mach numbers 0.3 and 0.7

\section{Conclusions}

An accurate and efficient numerical method for the solution of unsteady Navier-Stokes equations was presented in Section 3 for general systems of viscous conservation laws. The method is an extension of the numerical residual distribution schemes (RDS), which was originally developed to treat steady problems and has been adapted here to simulate unsteady viscous flows; such extension is new in the literature, to our knowledge. The unsteady RDS requires the numerical solution of a non-linear system of equations, where for consistency, the temporal mass matrix is constructed taking into account the upwinding properties of the spatial discretization scheme.

In order to check the accuracy of the method, the numerical simulation of the compressible, fully non-linear, non-parallel, twodimensional flow in the boundary layer in a $2 \mathrm{D}$ boundary layer over a flat plate at zero incidence has been considered. This test case bears quite stringent requirements on the numerical tool, in connection with numerical viscosity and stability properties. Moreover, comparison was made in terms of the small perturbations associated with the Tollmien-Schlichting (TS) waves, which made this test case a fairly demanding one.

The numerically obtained solutions were compared with the parallel, linear approximation, which was obtained solving the associated set of ordinary differential equations using a shooting method plus continuous orthonormalization, as explained in Section 4. Implementation of the method for the boundary layer problem was made in Section 5, where a computational domain was considered that accounts for a piece of the boundary layer in the streamwise direction, with appropriate boundary conditions for the incoming and outgoing flows; non-reflecting boundary conditions were implemented to match the unperturbed flow at free stream without non-physical effects. TS-like perturbations were generated 


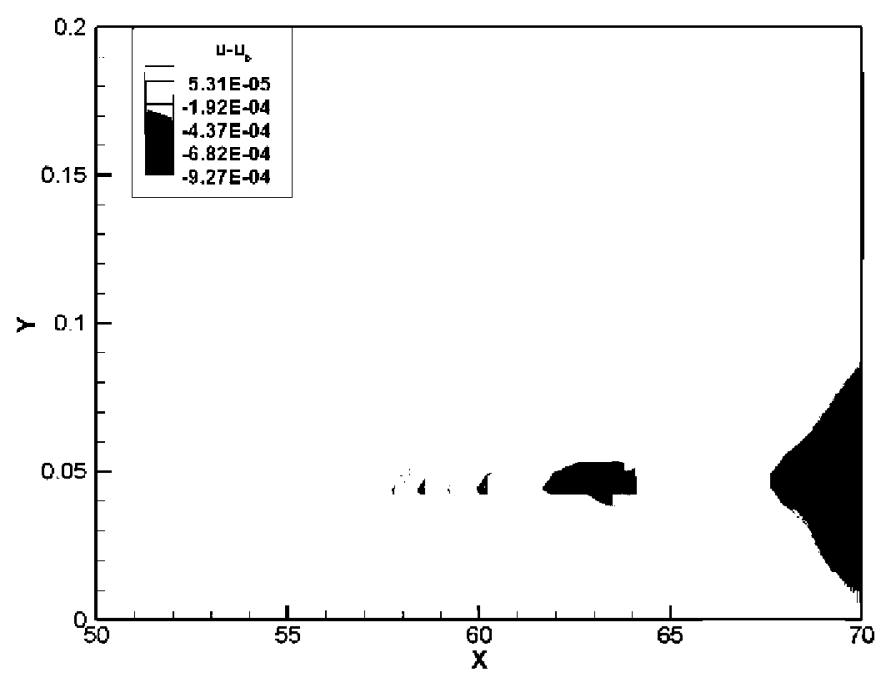

Fig. 12. Numerical differences between parallel and non-parallel steady solutions ( $M=0.7$ test case). Computational domain only partially showed.

near the upstream boundary using a vibrating membrane analogy.

Comparison with the analytically obtained solutions always showed a very good agreement, in spite of the quite demanding nature of the selected test problem. Thus, we expect that the method presented in this paper be useful in the efficient simulation of related compressible, viscous problems.

It is also worth to mention that, although this numerical test has been performed in a simple geometry. The application of this methodology to complex flows is straightforward and can be particularly suitable for aeroacoustic or Large Eddy Simulation (LES).

\section{Appendix A. Preliminary tests and convergence analysis}

Before proceeding with the comparative analysis, some preparatory work must be done.

To begin with, we calculate the unperturbed steady solution used in the numerical simulations, which includes non-parallel effects and is necessary to apply the boundary conditions (28). The iterative process to obtain such steady solution is initiated using the parallel Blasius compressible steady state at the streamwise position $x_{0}$ (see Eqs. (22)-(23)). This initial guess is obtained using an implicit Euler method with a large temporal step (CFL number equivalent to $10^{5}$ ) to avoid the growth of any temporal disturbances that can appear in the convergence process. The differences with the Blasius solution due to non-parallel effects are plotted in Fig. 12. As was to be expected (and remarked in Section 1), nonparallel effects are localized near the wall.

To obtain a pure TS-wave, it must be triggered introducing suitable disturbances in the computational domain. This agrees with the convectively unstable character of the laminar flat plate boundary layer, see Huerre and Monkewitz [10]. Thus, a very small perturbation is introduced at the upstream end of the computational domain. Two values of the perturbation amplitude were initially tested, namely $\varepsilon=10^{-10}$ and $\varepsilon=10^{-5}$, obtaining results that were almost identical. Thus, only disturbances with an amplitude $\varepsilon=10^{-10}$ were considered in the computations below.

As a second preparatory step, we calibrate the appropriate number of nodes in the streamwise $(n x)$ and wall-normal $(n y)$ directions, and time step $(\Delta t)$ to be used in the computations. To this end, a preliminary convergence study has been performed. In the considered test case, the converged steady solution is disturbed with a small perturbation of frequency $\omega=1$. Following the

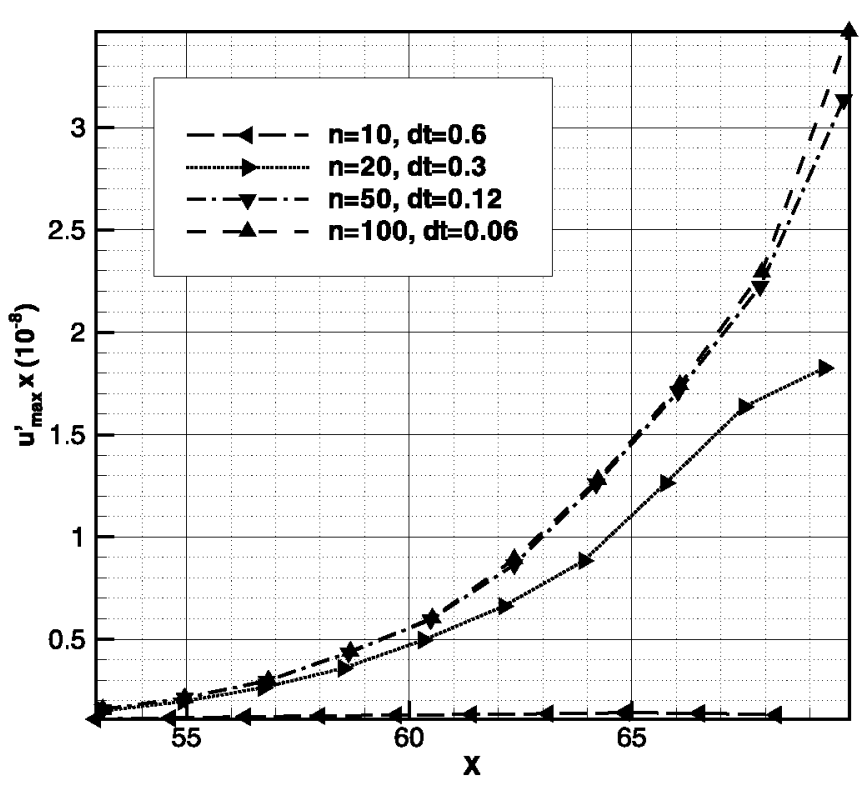

Fig. 13. Temporal convergence analysis for $\omega=1, \operatorname{Re}=10^{5}, M=0.7, x_{0}=50, n x \times$ $n y=125 \times 41$.

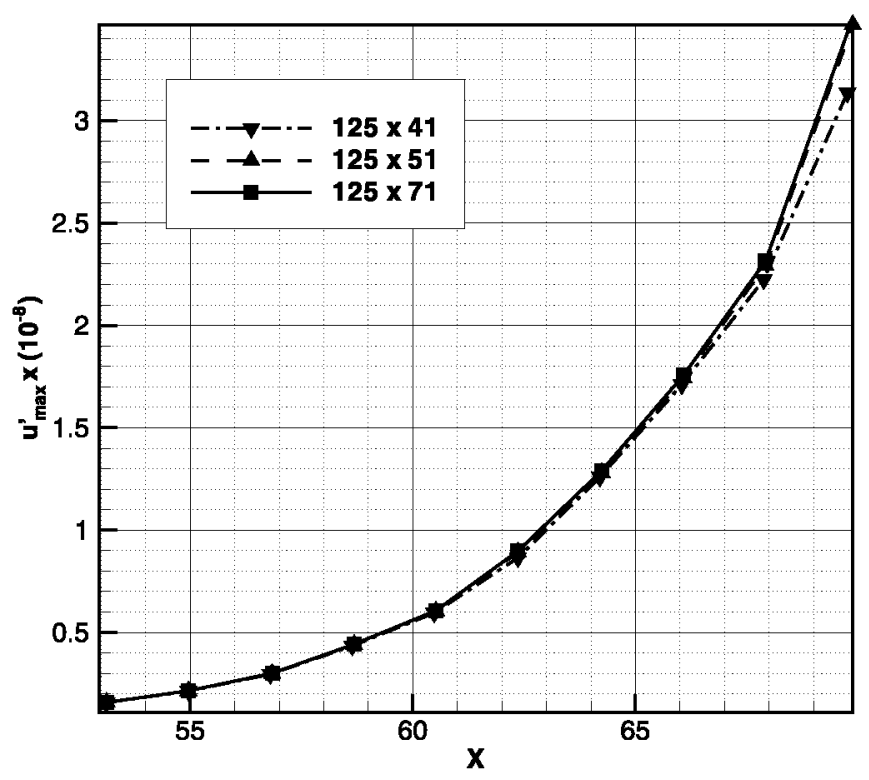

Fig. 14. Spatial convergence analysis in normal direction for $\omega=1, \operatorname{Re}=10^{5}, M=$ $0.7, x_{0}=50, n x=125$ and 100 steps per period.

methodology described in (29), such a disturbance is introduced at $x=50+\lambda_{1}$, namely near the upstream end of the selected computational domain. For the selected unstable TS-modes, the computational domain corresponds to $x \in[50,70]$. The wavelength $\lambda_{1}$ is extracted from Fig. 4 for both $M=0.3$ and $M=0.7$ at $x=50$. Since the phase velocity $c_{r}=\omega / \alpha_{r}=\omega \lambda /(2 \pi) \sim 0.3$ and the domain length is 20 , the excited wave needs around 70 time units to cross the computational domain. Thus, a slightly larger computational time interval, of 80 units has been selected. In all cases, spatial nodes are equi-distributed streamwise and follow the potential law

$x_{i}=i \cdot n x, \quad i=1, n x$

$y_{j}=y_{0} \cdot \frac{1-F^{(j-1)}}{1-F^{(n y-1)}}, \quad F=1.12, \quad j=1, n y$ 


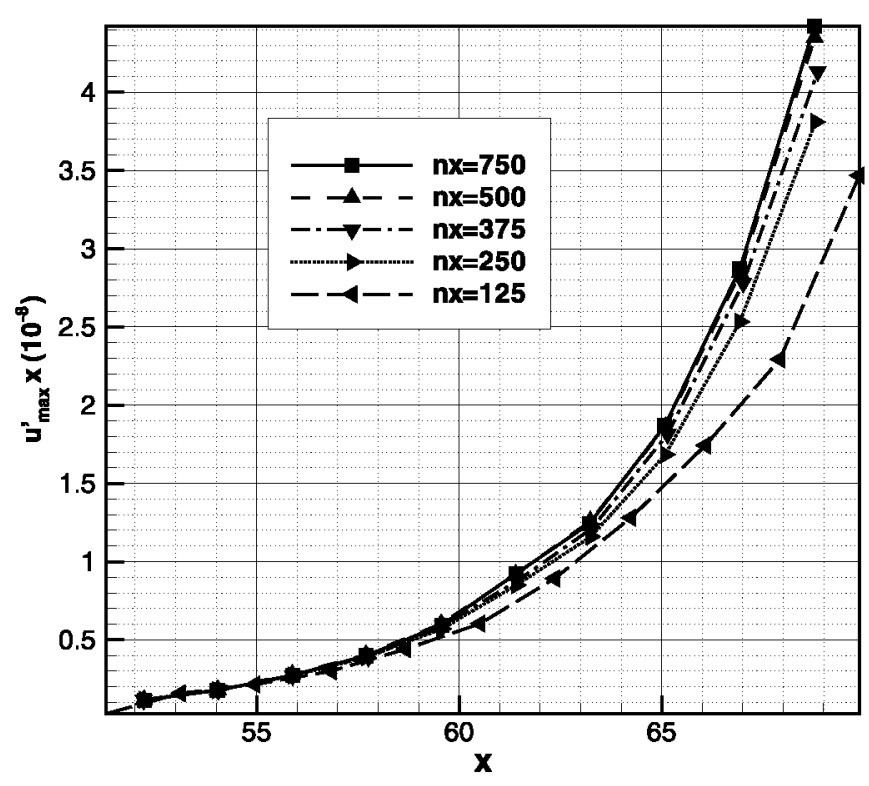

Fig. 15. Spatial convergence analysis in streamwise direction for $\omega=1, \operatorname{Re}=10^{5}$, $M=0.7, x_{0}=50, n y=51$ and 100 steps per period.

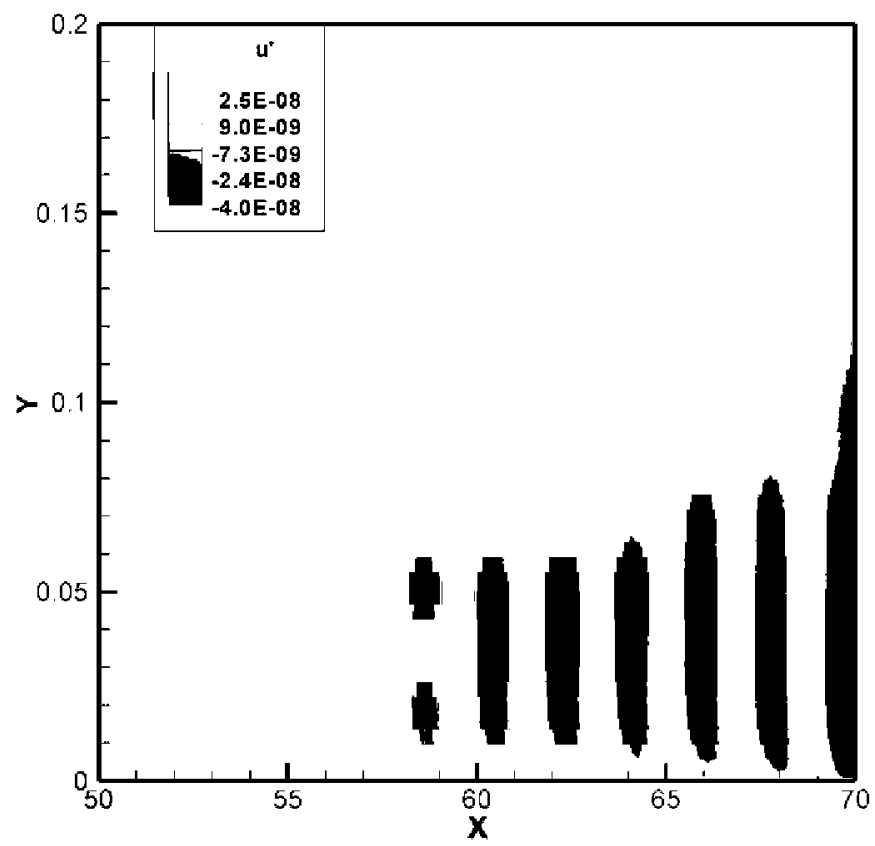

Fig. 16. Numerical solution of the perturbation velocity for $\omega=1, \operatorname{Re}=10^{5}, M=$ $0.7, x_{0}=50$. Computational domain only partially showed.

along the wall-normal direction. Note that, although the mesh is structured, RDS is designed also to be used in non-structured meshes. Thus, a similar sensibility analysis can be performed in more complex geometries.

The above mentioned convergence analysis has been performed in three consecutive steps:

(1) Temporal convergence: An initial mesh of $n x \times n y=125 \times 41$ has been considered. The temporal convergence has been analyzed by choosing time steps according to the law $\Delta t=$ $2 \pi /\left(\omega_{1} n\right)$, where $\omega_{1}$ is the TS-wave frequency and $n$ is the number of time steps per period. The perturbation maxima $u_{\max }^{\prime}$ for various values of $n$ are shown in Fig. 13. Although no significant differences are appreciated beyond 50 points per period, the value $n=100$ will be used in all computations be- low. Even for this high number of points, the final time step is only about 0.06 time units. This number of time steps is roughly a half of those used by Fasel and Konzelmann [7] in their incompressible boundary layer computations.

(2) Wall normal convergence: In order to check the effect of the number of points in the wall normal direction $y$, three different values of $n y$ are considered, namely 41,51 , and 71 . These node numbers correspond to characteristic variable increments in boundary layer units, $\Delta y^{+}=2,0.5$, and 0.05 . The temporal step $\Delta t$ and the number of nodes in the streamwise direction $n x=125$ do not vary. It can be observed in Fig. 14 that no further improvement results as $\Delta y^{+}$is lower than 1 . The value $n y=51$ has been selected for the comparative analysis.

(3) Streamwise convergence: With the values of $\Delta t$ and $n y$ selected above, the evolution of the perturbation maxima $u_{\max }^{\prime}$ for various values of $n x$ is given in Fig. 15, where it can be seen that results are very sensible to the streamwise refinement of the computational domain. Higher values of $n x$ imply a more accurate computation of spatial waves and, consequently, more accuracy in the resulting spatial amplification. Various values of $n x$ have been checked and, as a result, the number of points in the streamwise direction has been finally fixed as $n x=500$, which means that $\Delta x=0.04$. Such a spatial increment is approximately of the order of the displacement boundary layer thickness, in boundary layer scale $\Delta x^{+} \simeq 40$. Although this value is slightly smaller than that used by Rai and Moin [15] for the case $M=0.0849$ ( $\Delta x^{+} \simeq 25$ ), it involves the use of larger number of points per wavelength than those used by Fasel and Konzelmann [7], in their computations of the incompressible case with a spatial fourth order scheme. As the upwinding nature of the RDS scheme introduces more numerical viscosity than the centered schemes used in incompressible flows, it is expected that a larger number of nodes per wavelength are needed here. However, centered schemes, although more appropriate for these viscous problems, are not numerically stable in compressible flows.

Summarizing, the selected number of steps and nodes are: 100 steps per time period, $n y=51$, and $n x=500$. With this selection, a typical numerical solution of the $u^{\prime}$ disturbance for $\omega=1$ at $M=0.7$ is shown in Fig. 16 .

\section{References}

[1] R. Abgrall, Towards the ultimate conservative scheme: Following the quest, J. Comput. Phys. 167 (2001) 277-315.

[2] D.A. Caraeni, Development of a multidimensional upwinding residual distribution solver for large eddy simulation of industrial turbulent flows, Ph.D. thesis, Lund Institute of Technology.

[3] D. Caraeni, M. Caraeni, L. Fuchs, A parallel multidimensional upwind algorithm for LES, AIAA-2001-2547.

[4] A. Csik, H. Deconinck, Space-time residual distribution schemes for hyperbolic conservations laws on unstructured linear finite elements, Int. J. Numer. Methods Fluids 40 (3) (2002) 573-581.

[5] A. Davey, An automatic orthonormalization method for solving stiff boundaryvalue problems, J. Comput. Phys. 51 (1983) 343-356.

[6] H. Deconinck, M. Ricchiuto, Residual Distribution Schemes: Foundation and Analysis, Encyclopedia of Computational Mechanics, vol. 3, John Wiley and Sons, 2007.

[7] H. Fasel, U. Konzelmann, Non-parallel stability of a flat-plate boundary layer using the complete Navier-Stokes equations, J. Fluid Mech. 221 (1990) 311347.

[8] A. Ferrante, H. Deconinck, Solution of the unsteady Euler equations using residual distribution and flux corrected transport, Technical Report VKI-PR 97-08.

[9] M. Gaster, On the effects of boundary-layer growth on flow stability, J. Fluid Mech. 66 (3) (1974) 465-480.

[10] P. Huerre, P.A. Monkewitz, Global instability analysis, Annu. Rev. Fluid Mech. 22 (1990) 473-537.

[11] H. Keller, Numerical Solution of Two Point Boundary Value Problems, CBMSNSF Regional Conferences in Applied Mathematics, vol. 24, SIAM. 
[12] U. Konzelmann, U. Rist, H. Fasel, Erzeugung dreidimensionaler, rumlich angefachter Strwellen durch periodisches Ausblasen und Absaugen in einer Plattengrenzschichtstrmung, ZAMM 67 (5).

[13] L. Mack, Boundary-layer linear stability theory, AGARD R-709 (1984) 153-170.

[14] H. Paillere, Multidimensional upwind residual distribution schemes for the Euler and Navier-Stokes equations on unstructured grids, $\mathrm{PhD}$ thesis, Universite Libre de Bruxelles, Belgium.

[15] M.M. Rai, P. Main, Direct numerical simulation of transition and turbulence in a spatially evolving boundary layer, J. Comput. Phys. 109 (1993) 169-192.

[16] M. Ricchiuto, A. Csik, H. Deconinck, Residual distribution for general timedependent conservation laws, J. Comput. Phys. 209 (2005) 249-289.
[17] W.S. Saric, A.H. Nayfeh, Nonparallel stability of boundary-layer flows, Phys. Flu ids 18 (1975) 945.

[18] H. Schlichting, K. Gersten, Boundary Layer Theory, Springer-Verlag, Berlin, 2000

[19] F. Smith, On the high Reynolds number theory of laminar flows, IMA J. Appl. Math. 28 (1982) 207-281.

[20] V. Theofilis, Numerical experiments on the stability of dimensional linear study leading edge boundary layer flow: a two-dimensional linear study, Int. J. Numer. Methods Fluids 16 (1993) 153-170.

[21] E. Valero, J. de Vicente, G. Alonso, The application of compact residual distribution schemes to two-phase flow problems, Comput. Fluids 38 (2009) 1950 1968. 